\title{
Spontaneous magnetization in heterometallic NiFe-MOF-74 mi- croporous magnets by controlled iron doping
}

\author{
Víctor Rubio-Giménez ${ }^{\mathrm{a}}$, Joao C. Waerenborgh ${ }^{\mathrm{b}}$, Juan M. Clemente-Juanª and Carlos Martí-Gastaldo ${ }^{\text {a* }}$ \\ ${ }^{a}$ Instituto de Ciencia Molecular (ICMol), Universitat de València, Catedrático José Beltrán 2, 46980, Paterna (Spain) \\ bentro de Ciências e Tecnologias Nucleares, Instituto Superior Técnico, Universidade de Lisboa, 2695-066 Bobadela LRS (Portu- \\ gal) \\ E-mail: carlos.marti@uv.es
}

\begin{abstract}
We report the direct synthesis of mixed-metal NiFe-MOF-74 solids that display combination of porosity with ferrimagnetic ordering. Compared to the undoped Ni phase, controlled doping with Fe enables to modify intra and interchain magnetic interactions for the onset of spontaneous magnetization at temperatures fixed by the doping level. Synthesis of porous magnets remains somewhat elusive due to the difficulties in isolating foreseeable metal-organic architectures that combine small bridging linkers, for strong magnetic coupling, with polyaromatic connectors responsible for porosity. In turn, we demonstrate that metal doping is better fitted to modify the magnetism of MetalOrganic Frameworks already available simply by suitable choice of their nature and relative ratio in isostructural solid solutions.
\end{abstract}

One of the most fruitful approaches for the development of advanced functional materials relies on the combination of two or more functions in a single solid. This can result in mere coexistence or in interaction between simple functions, for synergetic or cooperative effects. Among other families of multifunctional materials, ${ }^{1-3}$ Metal-Organic Frameworks (MOFs) featuring combination of magnetism and micro/nano-porosity have attracted important attention based on the interplay of the magnetic properties intrinsic to the organic/inorganic framework with the potential guests that can be accommodated in the empty space available. This can result in sophisticated materials of interest in sensing, magnetic sequestration or as stimuli-responsive solids. ${ }^{4-7}$ Engineering of long-range magnetism in MOFs is somewhat challenging though, as the organic polyaromatic linkers generally used for directing the assembly of metal-oxo clusters into porous architectures mediates weak magnetic exchange between spin carriers. This is why the vast majority of magnetic MOFs reported features combination of an inorganic sub-network of variable dimensionality ( 1,2 or $3 \mathrm{D})$, built by bridging metal centers with small inorganic molecules like $\mathrm{O}_{2}{ }^{-}, \mathrm{OH}^{-}, \mathrm{PO}_{4}{ }^{3-}$ or $\mathrm{CN}^{-}$to mediate strong enough magnetic coupling, and an organic one that is responsible for controlling overall porosity. ${ }^{6}$

Discovery of new porous frameworks with foreseeable magnetic properties is thus a difficult task, as the latter will be dictated by how the inorganic (magnetic) and organic (porous) networks are interconnected in the final structure, which is often difficult to predict. In turn, overall magnetism can be more easily manipulated by an adequate choice of the metal centers in the inorganic network for a given structure, as this will modify the nature and extent of the magnetic exchange according to the Goodenough-Kanamori rules. This approach has been nicely illustrated for the isostructural family of porous $3 \mathrm{D}$ magnets $\mathrm{M}_{3}(\mathrm{HCOO})_{6}(\mathrm{M}=\mathrm{Mn}, \mathrm{Fe}, \mathrm{Co})$, in which magnetic ordering can be shifted between $2-16 \mathrm{~K}$ depending on the metal of choice. ${ }^{8-10}$ In turn, the spin-canted antiferromagnetic (AF) ordering of $\mathrm{Ni}_{18}\left[\left(\mathrm{HPO}_{4}\right)_{14}(\mathrm{OH})_{3} \mathrm{~F}_{9}\left(\mathrm{H}_{3} \mathrm{O} / \mathrm{NH}_{4}\right)_{4}\right]$ (also known as
VSB-1) at $\mathrm{T}_{\mathrm{C}}=10.5$ can be transformed into ferrimagnetic ordering at $20 \mathrm{~K}$ simply by doping with $10 \%$ of $\mathrm{Fe}$ (III). ${ }^{11}$ This magnetic versatility coupled to the recent advances in post-synthetic metal exchange ${ }^{12}$ offer an appealing alternative to the synthesis of new magnetic MOFs.

The MOF-74 family (also known as CPO-27) with formula $\mathrm{M}_{2}$ (dhtp) $\left(\mathrm{H}_{2} \mathrm{O}\right)_{2}(\mathrm{M}=\mathrm{Mg}, \mathrm{Mn}, \mathrm{Fe}, \mathrm{Co}, \mathrm{Ni}, \mathrm{Zn}$; dhtp=2,5-dihydroxyterephthalate) ${ }^{13,14}$ is ideal to prove the effects of metal doping over the magnetic properties of the solid. These 3D solids are built from the interconnection of $\mathrm{M}\left(\mu_{2}-\mathrm{O}\right)_{4}(\mathrm{O})_{2}$ chains with dhtp to produce a honeycomb lattice with one-dimensional channels that are filled with solvent molecules (Figure 1a). According to previous studies, pure $\mathrm{Co}, \mathrm{Fe}$ and Ni MOF-74 phases display weak ferromagnetic (FM) intrachain interactions that coexist with AF interactions between neighboring chains at lower temperatures. ${ }^{15,16}$ This results in no spontaneous magnetization above $2 \mathrm{~K}$ due to the formation of an antiferromag-

netic ground state. We report the onepot synthesis of mixed-metal NiFe-MOF-74 solids that display combination of porosity with ferrimagnetic ordering. Doping with $\mathrm{Fe}(\mathrm{III})$ at variable atomic $\%$ permits modulating the onset of spontaneous

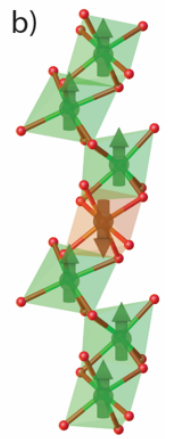

Figure 1. a) Structure of MOF-74 along the $c$ axis. Accessible porosity is represented by $1 \mathrm{D}$ Connolly surfaces in blue. b) Doping of $\mathrm{M}\left(\mu_{2}-\mathrm{O}\right)_{4}(\mathrm{O})_{2}$ chains with $\mathrm{Fe}(\mathrm{III})$ for introduction of antiferromagnetic interactions. Nickel (green), iron (orange). 
magnetization between 10 and $16 \mathrm{~K}$, due to controlled introduction of AF interactions across the metal-oxo chains (Figure $1 \mathrm{~b}$ ) for variable interchain coupling, while retaining the original porosity for low doping levels. We also explore the effects of guest sorption over the magnetism of these solids.

NiFe-MOF-74 solids were prepared under anaerobic conditions by reaction of variable amounts $\mathrm{Ni}\left(\mathrm{NO}_{3}\right)_{2}$ and anhydrous $\mathrm{FeCl}_{2}$ with $\mathrm{H}_{4} \mathrm{dhtp}$ in a mixture of DMF and $\mathrm{MeOH}$ in a glove box by following a reported procedure with slight modifications (synthetic details in SI2 $)^{17}$. Solids were isolated as green, microcrystalline powders. Exposure to open air results in gradual color change to dark green as result of iron oxidation in the presence of atmospheric $\mathrm{O}_{2}$. Air sensitivity of $\mathrm{Fe}^{\mathrm{II}}$-MOF74 has been previously documented. ${ }^{17}$ Note that direct incorporation of $\mathrm{Fe}$ (III) to the structure of MOF74 is not recommendable as it might lead to a different phase based on charge balance considerations and hard Lewis character of this cation. We opted for the gradual oxidation of metal centers already incorporated to the framework instead, as it is more likely to be respectful with the structure originally formed. Morphology and crystal habit were studied by Scanning Electron Microscopy (SEM) and compared to undoped Ni-MOF-74 synthesized under equivalent conditions. All three solids are composed of micrometric crystals, but the increase in iron content produces crystals with a smaller particle size and a rounder shape (Figure SI1). There is no obvious effect of iron doping levels over particle size. Total metal content was analyzed by Inductively Coupled Plasma Optical Emission Spectroscopy (ICP-OES) after digestion of as-made solids in $\mathrm{HNO}_{3}$ (Table SI1). Analysis is consistent with the nominal ratio of metals in the synthetic gel thus confirming quantitative loading of iron from solution for the formation of $\mathrm{Ni}_{1.96} \mathrm{Fe}_{0.04}$ (1) and $\mathrm{Ni}_{1.6} \mathrm{Fe}_{0.4}$-MOF-74 (2). Metal distribution was analyzed by mapping different regions of the solids with X-ray Energy Dispersive Spectroscopy (EDS). Data confirms the incorporation of $\mathrm{Ni}$ and $\mathrm{Fe}$ to the structure with a small statistic deviation in the Ni:Fe ratio of $54.0 \pm 5.4$ (1) and $3.4 \pm 0.3$ (2). This is indicative of a homogeneous distribution of metals for different crystals of the sample and rules out metal clustering at a micrometric level or the formation of segregated phases (Figure SI2). Previous studies for more complex multi-metallic MOF-74 samples have shown more heterogeneous distributions. ${ }^{18} \mathrm{We}$ ascribe this difference to the use of $\mathrm{Ni}$ and $\mathrm{Fe}$ (II) cations that are chemically more alike. Structure and phase purity of the solids was confirmed with a Pawley refinement of the powder X-ray diffraction (PXRD) data collected at room temperature by using the single-crystal data available for the Ni-MOF-74 phase as initial guess. ${ }^{19}$ Full-profile fits yield acceptable agreement factors and profile differences for $\mathbf{1}$ and $\mathbf{2}$ (Figure 2). In comparison with the $100 \% \mathrm{Ni}$ phase, refined unit cell parameters follow a slight contraction with increasing iron loading, consistent with the partial replacement of $\mathrm{Ni}(\mathrm{II})$, ionic radius of 0.69 $\AA \AA^{20}{ }^{20}$ with smaller Fe (II) (cf. 0.61). Besides cell contraction, thermogravimetric analysis suggests that iron doping has a negative effect over thermal stability. $\mathbf{1}$ and $\mathbf{2}$ decompose at lower temperatures that the undoped material (Figure SI7).

Solids were activated under dynamic vacuum $\left(10^{-3} \mathrm{mbar}\right)$ at 200 ${ }^{\circ} \mathrm{C}$ for 24 hours. Porosity was studied by $\mathrm{N}_{2}$ adsorption-desorption measurements at $77 \mathrm{~K}$ (Figure 3). 1 displays type-I reversible $\mathrm{N}_{2}$ uptake with no hysteresis, characteristic of a microporous material. Calculated multi-point BET of $1111 \mathrm{~m}^{2} \cdot \mathrm{g}^{-1}$ and total pore volume of $0.53 \mathrm{~cm}^{3} \cdot \mathrm{g}^{-1}$ are slightly below the value reported for Ni-MOF- $74^{19}$ likely due to differences in the activation protocol. In turn, $\mathbf{2}$ features a type-I isotherm in the low-P region with a small hysteresis loop at

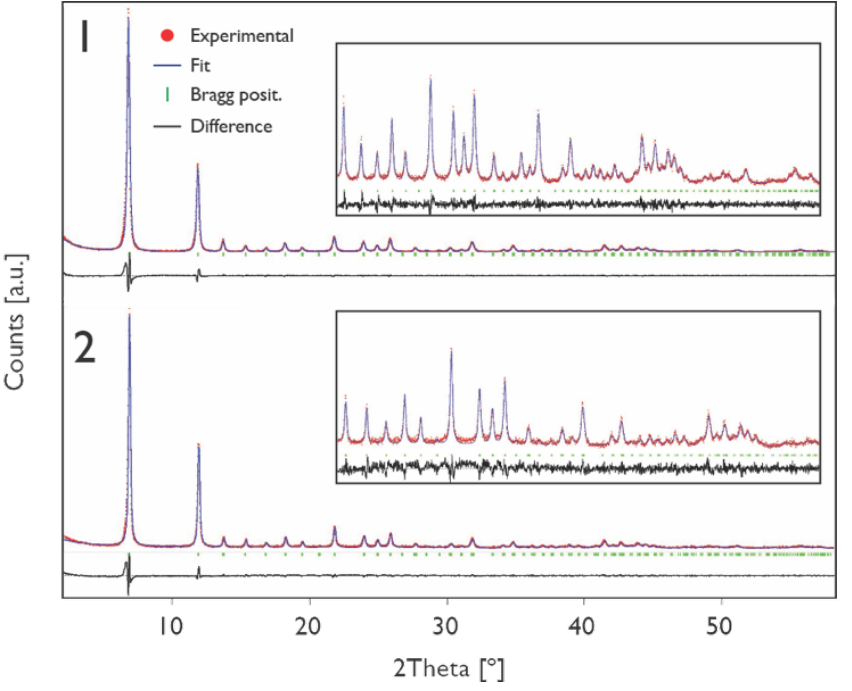

Figure 2. Pawley refinement of PXRD of 1 and 2 showing the experimental (red points), calculated (blue), difference patterns (black) and Bragg positions (green). Inset is a zoom-in of the high-angle region. See Table SI 2 for refined unit cell parameters and agreement factors.
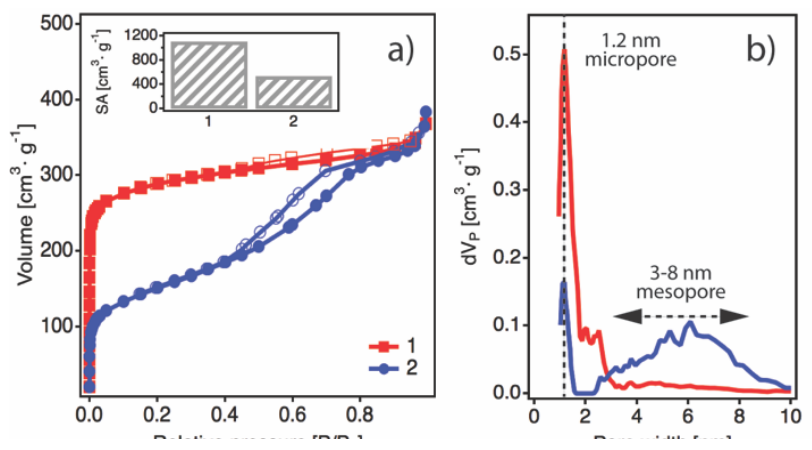

Figure 3. a) $\mathrm{N}_{2}$ isotherms and BET surface areas for $\mathbf{1}$ (red) and $\mathbf{2}$ (blue). b) Pore size distribution. See SI3 for more details.

$0.5<\mathrm{P} / \mathrm{P}_{0}<0.8$ more characteristic of a type-IV mesoporous material for a drastic reduction in BET of $537 \mathrm{~m}^{2} \cdot \mathrm{g}^{-1}$ and pore volume of $0.52 \mathrm{~cm}^{3} \cdot \mathrm{g}^{-1}$. Analysis of the pore size distribution (PSD) by using non-linear solid density functional theory (NLDFT) indicates that microporosity is dominated by a mean pore diameter of $11.7 \AA$ in both solids, in good agreement with the value of $11.85 \AA$ calculated from the structure. However, 2 also displays a broad dispersion of mesopores between 3 and $8 \mathrm{~nm}$ (Figure $3 \mathrm{~b}$ ). We ascribe these features in the pore size distribution to intergrain mesoporosity caused by the smaller and more irregular particles due to the increasing iron content in $\mathbf{2}$. Note that isotherms were collected for solids after exposure to open air. This can result in gradual oxidation of $\mathrm{Fe}$ (II) centers in the MOF structure which shall trigger in incorporation of anions like chloride, present originally in the reaction medium, for overall charge balance. This was confirmed by ICP-OES analysis of the solids after gas sorption experiments, which reveals a concomitant increase on the amount of chloride with iron doping of $\leq 0.4$ and $4.9 \%$ for 1 and 2, respectively (Table SI1). Next, we used high-resolution X-Ray Photoelectron Spectroscopy (XPS) to confirm the chemical states of metals and chloride (SI4). Ni $2 p$ spectra can be fitted to four components by using only the $2 \mathrm{p}_{3 / 2}$ portion of the spectrum and agrees well with the presence of $\mathrm{Ni}$ (II) with the most in- 
tense emission line centered around $856 \mathrm{eV} .{ }^{21} \mathrm{Fe} 2 \mathrm{p}$ signal can overlap strongly with the Ni LMM Auger peaks which hinders quantification of the Ni:Fe ratio. The poor amount of iron in sample coupled to the small value of the Scofield's relative sensitivity factor in very poor signal to noise ratio for the XPS spectrum of 1 which renders very difficult analysis. In turn, for 2 a broad $\mathrm{Fe} 2 \mathrm{p}_{3 / 2}$ appears above $712 \mathrm{eV}$ that suggests $\mathrm{Fe}(\mathrm{III})$ is the dominant specie. ${ }^{21} \mathrm{Cl} 2 \mathrm{p}$ spectra features only $2 \mathrm{p}_{3 / 2}$ and $2 \mathrm{p}_{1 / 2}$ lines with a spin-orbit coupling close to $1.6 \mathrm{eV}$ that confirms the presence of chloride anions in the solid. We also used far-IR spectroscopy to confirm the role played by chloride anions in the structure of NiFe-MOFs. As shown in Figure SI6, comparison of the $100 \% \mathrm{Ni}$ phase with 2 confirms the appearance of a new vibration mode at $389 \mathrm{~cm}^{-1}$ that agrees well with the expected stretching frequency for $\mathrm{Fe}(\mathrm{III})-\mathrm{Cl}^{22}$

Mössbauer spectroscopy is more adequate to probe the electronic structure of iron centers. The small doping in $\mathbf{1}$ prevented analysis of this sample. In turn, spectra of $\mathbf{2}$ were collected between 30 and 295 $\mathrm{K}$. As shown in Figure 4, there is two quadrupole doublets that can be assigned to the co-existence of $\mathrm{Fe}(\mathrm{III})$ and $\mathrm{Fe}(\mathrm{II})$ metal sites (SI5). The sub-spectrum with higher intensity has an isomer shift (IS) relative to metallic $\alpha$-Fe at room temperature typical of highspin $\mathrm{Fe}$ (III) octahedrally coordinated by oxygen atoms. ${ }^{23}$ However, none of the absorption peaks of this doublet can be adequately fitted by single lorentzian lines. This is likely due to the different environments of the $\mathrm{Fe}$ (III) cations that arise from the statistical distribution of $\mathrm{Fe}$ (III), $\mathrm{Fe}$ (II) and $\mathrm{Ni}$ (II) cations in the lattice. Different environments imply slightly different hyperfine interactions. The slight differences in IS and quadrupole splitting (QS) values are not large enough to give rise to resolved peaks. Accordingly, absorption peaks were better fitted by using a distribution of lorentzians. The subspectrum with lower intensity has an IS typical of high-spin Fe(II). The high IS value is also consistent with octahedral coordination by oxygen atoms. The lower, temperature independent QS of Fe(III) and the higher QS of Fe(II), that increases with decreasing temperature, confirm the assignments of the Mössbauer sub-spectra to these Fe oxidation states. The increase of IS values with decreasing temperature is linked to the second order Doppler shift. According to our analysis, $10 \pm 2 \%$ of the $\mathrm{Fe}$ atoms are in the $2+$ oxidation state whilst the remaining $\mathrm{Fe}$ is in the $3+$ oxidation state. It is also worth noting that the spectra at $4 \mathrm{~K}$ (SI5) shows a significant broadening of the absorption peaks and an additional broad absorption band in the velocity range $-4.5,5 \mathrm{~mm} / \mathrm{s}$ suggesting a significant slowdown of the thermally induced fluctuations of the Fe magnetic moment directions when compared to the frequencies typical for paramagnetic states as those observed at $30 \mathrm{~K}$ and above. This is consistent with the occurrence of long-range magnetic correlations below 9 $\mathrm{K}$, typical of an ordered magnetic state.

Magnetic properties were studied by using ground polycrystalline solids. We also measured the undoped Ni-MOF-74 phase to highlight more clearly the changes associated to iron doping. As shown in Figure 5a, thermal variation of $\chi \mathrm{T}$ follows a different trend linked to variable iron doping. Ni-MOF-74 displays a slight increase in $\chi \mathrm{T}$ upon cooling, down to low$\mathrm{T}$ where it becomes more abrupt and defines a maximum at 20 $\mathrm{K}$ followed by a steep fall that may be associated to AF ordering.

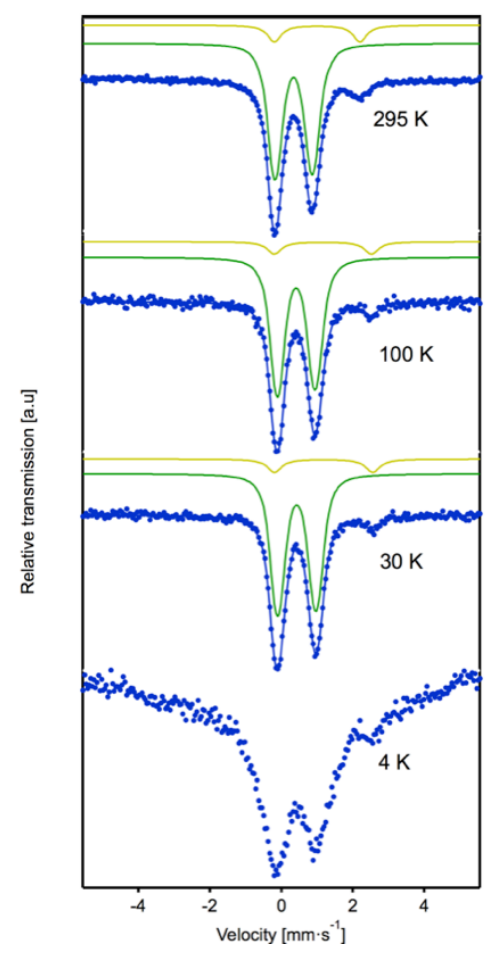

Figure 4. Mössbauer spectra of 2 collected at different temperatures showing $\mathrm{Fe}(\mathrm{II})$ (yellow) and $\mathrm{Fe}$ (III) (green) doublets. Low Fe content in 1 prevented a similar analysis.

This suggests combination of weak intrachain FM interactions between $\mathrm{Ni}$ (II) sites alongside weaker AF interactions between neighbouring chains operating at lower temperatures. 1 behaves similarly at high temperatures but displays a minimum at $17 \mathrm{~K}$, characteristic of additional intrachain AF interactions. The effect of iron doping can be more clearly seen in 2 , which displays a continuous decrease in $\chi \mathrm{T}$ with cooling, down to a minimum at $7 \mathrm{~K}$. Below these minima, $\chi \mathrm{T}$ of both, 1 and 2 , reaches a maximum that corresponds to an abrupt jump in the magnetic susceptibility (SI6) suggesting the onset of long-range magnetic ordering. Fit of the susceptibility data in the paramagnetic regime to the Curie-Weiss law yields Curie constant values (C) of $1.99(1) \mathrm{emu} \cdot \mathrm{K} \cdot \mathrm{mol}^{-1}$ for 1 and $2.97(5) \mathrm{emu} \cdot \mathrm{K} \cdot \mathrm{mol}^{-1}$ for 2 that agree well with the expected spin-only values for the sum of non-interacting $\mathrm{Ni}^{2+}(\mathrm{S}=1)$ and high-spin $\mathrm{Fe}^{3+}(\mathrm{S}=5 / 2)$ according to the formula $\mathrm{Ni}_{2-\mathrm{x}} \mathrm{Fe}_{\mathrm{x}}$ (dhtp) ( $\left.\mathrm{x}=0.04,0.4\right)$. Weiss constants $(\Theta)$ change from 5 for 1 to $-82 \mathrm{~K}$ for 2 . This is due to the introduction of
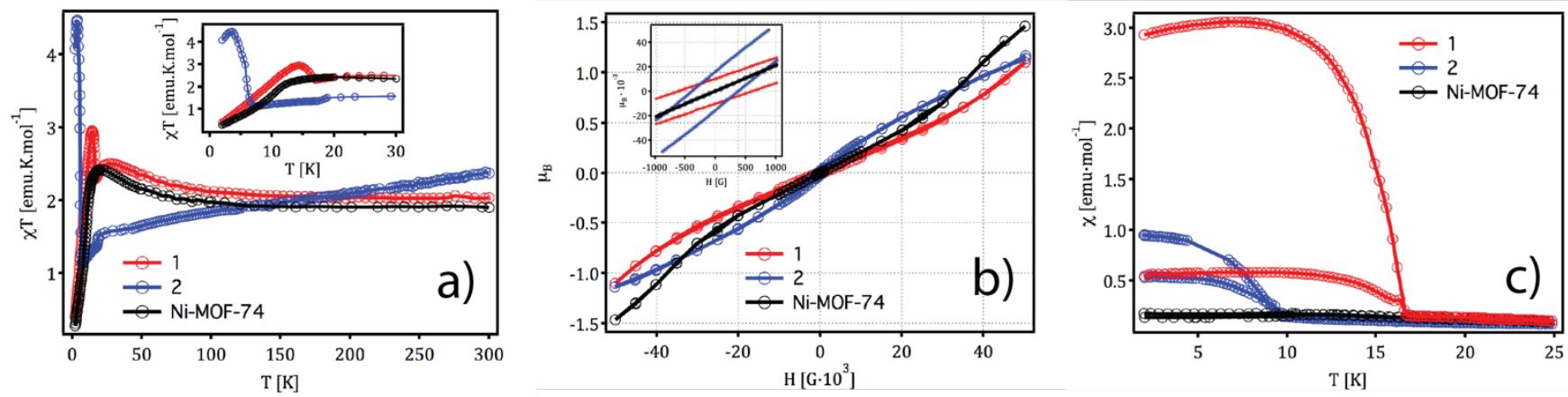

Figure 5. Static magnetic data of undoped Ni-MOF-74 (black), 1 (red) and 2 (blue). a) Variation of $\chi \mathrm{T}$ product with the temperature. b) Hysteresis loops collected at $2 \mathrm{~K}$. c) FC/ZFC measurements at $10 \mathrm{G}$ showing magnetic irreversibility below $\mathrm{T}_{\text {irr }}$ upon Fe doping. See SI6 for more details. 
a variable amount of iron centres that results in a statistical distribution of FM Ni-O-Ni and AF Ni-O-Fe pairs. For low doping the number of FM pairs is much higher than AF ones and susceptibility of 1 is overall dominated by the former. In turn, higher doping increases the ratio of AF pairs that results in dominant $\mathrm{AF}$ interactions according to the high, negative $\Theta$ value of 2 . Field dependence of the magnetization displays a linear increase up to small magnetization values far from saturation at $5 \mathrm{~T}$, typical of ferrimagnets (SI6). As shown in Figure 5b, unlike Ni-MOF-74, both solids display hysteresis loops at $2 \mathrm{~K}$ characteristics of magnetic ordering at low-T. Coercive fields are smaller than $0.1 \mathrm{~T}$, which qualifies NiFe-MOF-74 as soft magnets. Divergence in the field-cooled (FC)/zero-field-cooled (ZFC) measurements (Figure 5c) confirms magnetic irreversibility below $\mathrm{T}_{\text {irr }}=$ 16.4 and $9 \mathrm{~K}$ for $\mathbf{2}$ and 1, respectively. Magnetic ordering was also confirmed by ac dynamic susceptibility measurements in the presence of an external field of $3.95 \mathrm{G}$, oscillating between 1 and $100 \mathrm{~Hz}$ (Figure 6). Both solids display sharp maxima for the in-phase $\left(\chi^{\prime}\right)$ and out-of-phase $(\chi ")$ signals. Critical temperatures $\left(T_{\mathrm{C}}\right)$, defined as the temperature where $\chi$ " becomes nonzero, are consistent with static FC/ZFC measurements and decrease from 16.7 for 1 to 9.5 in 2. As depicted in Figure 7, changes in the magnetism upon iron doping can be rationalized qualitatively by considering its effect over the magnetic ground state of the $1 \mathrm{D}$ metal-oxo chains. For very low doping, chains in 1 can be described by random-sized FM Ni-O-Ni fragments separated by isolated iron centers for a low density of localized AF Ni-O-Fe pairs. This results in chains with high-spin ground states similar to the undoped solid, which agrees well with the similar maxima in the $\chi \mathrm{T}$ of 1 and Ni-MOF-74 at low-T (Figure 5a). In turn, increasing levels of doping will induce the formation of AF Fe-O-Fe chain segments across the chain in 2 . Whereas for odd number of iron centers the setting will be similar to low doping levels, even numbers will promote AF coupling between the Ni-O-Ni fragments. In general, this will lead to lower spin ground states for a continuous decrease in the magnetic susceptibility as observed for 2 . Compared to the effective cancelling of spins for no net magnetic signal in the AF ground state of the undoped phase, Fe doping enables coexistence of intrachain $\mathrm{F}$ and $\mathrm{AF}$ interactions across the magnetic chains. This precludes total cancelling and originate non-compensated magnetic moments responsible for the spontaneous magnetization at low-T observed for $\mathbf{1}$ and $\mathbf{2}$. We argue the higher spin ground state
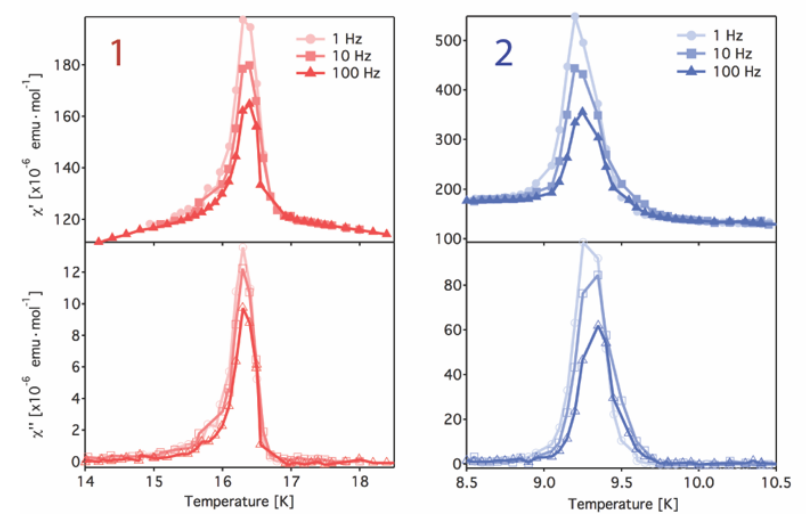

Figure 6. Ac dynamic susceptibility of $\mathbf{1}$ (left) and $\mathbf{2}$ (right) In-phase (top, filled symbols) and out-of-phase signals (bottom, empty symbols) at 1 (circle), 10 (square), and $100 \mathrm{~Hz}$ (triangle). configuration of the chain in 1 , as result of very low doping, might be responsible for its higher $T_{c}$ compared to 2 .

A recent work by Long and co-workers reveals that the magnetism
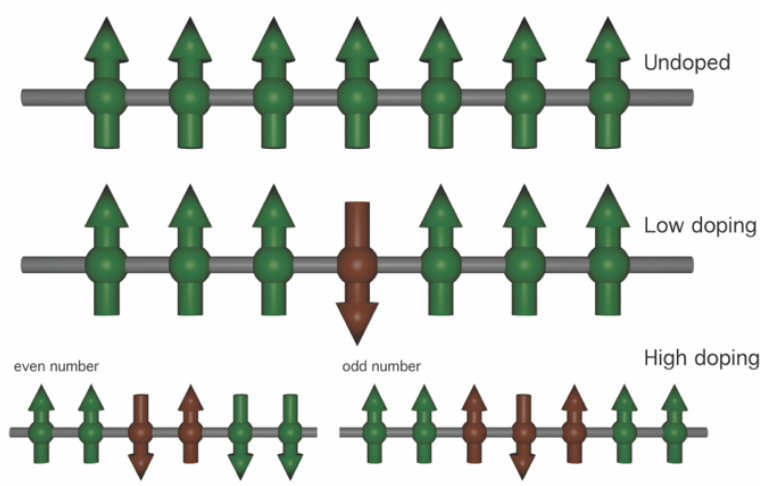

Figure 7. Proposed changes in the spin configuration of the magnetic chains in MOF-74 upon iron doping. Nickel (green), iron (orange).

of Fe-MOF-74 is sensitive to the guest. ${ }^{15}$ Loading of adsorbates with variable polarizability and affinity to interact with the framework enables modulation of the nature and extent of magnetic superexchange across the chains without modifying the electronic configuration of the metals. This is probably promoted by local perturbation of the electron density at the $\mathrm{Fe}$ (II) centers linked to guest interaction. ${ }^{24}$ Hypothetically, this same phenomenon might be exploited to induce a shift in the $T_{c}$ of ferrimagnetic 1 or 2 upon gas loading. Samples were transferred to a glass tube, cooled with liquid $\mathrm{N}_{2}$, loaded with $22 \mathrm{~cm}^{3}$ of $\mathrm{C}_{2} \mathrm{H}_{4}$ (approx. 14 bar) and sealed with flame. See SI7 for experimental details. Comparison of FC/ZFC magnetization curves for guest-free and guest-loaded samples suggests that variation of the magnetism remains almost negligible, with only minor changes to the $\mathrm{T}_{\text {irr }}$ of both solids (Figure SI7). We ascribe this behavior to the oxidation of close to $90 \%$ of the iron centers and concomitant chloride coordination for charge balance. $\mathrm{Fe}$ (II) vacant sites in Fe-MOF-74 are free and prone to interact with gas molecules. ${ }^{15} \mathrm{In}$ turn, choride anions in $\mathbf{1}$ and $\mathbf{2}$ might be blocking the coordination sphere of the metal, as suggested by far-IR spectroscopy, thus neglecting interaction with the guest for minimum influence on the magnetic properties.

In summary, one-pot synthesis of Ni-MOF-74 in the presence of iron enables controlled doping of isostructural, mixed-metal solid solutions. Compared to the undoped phase, NiFe-MOF-74 solids display combination of porosity and ferrimagnetic ordering as result of the modulation of the chain spin state for variable intra and interchain magnetic interactions. This induces the occurrence of spontaneous magnetization at variable temperatures that are controlled by the quantity of iron incorporated. Direct synthesis of porous magnets remains somewhat elusive due to the difficulties in isolating foreseeable metal-organic architectures that combine small bridging linkers, for strong magnetic coupling, with polyaromatic connectors responsible for porosity. In turn, metal doping of available MOF architecture is well fitted to engineering their magnetic behavior by chemical means, simply by rational combination of metals at variable ratios. We are confident the versatility of this approach will also benefit from the recent advances in post-synthetic metathesis of MOFs, ${ }^{12,25-27}$ that provides the synthetic tools necessary to design 
new magnetic materials not accessible by conventional synthesis, but by milder cation exchange reactions instead.

\section{ASSOCIATED CONTENT}

\section{Supporting Information}

The Supporting Information is available free of charge on the ACS Publications website.

Synthetic and experimental details. Physical characterization and supporting tables and figures (PDF).

\section{AUTHOR INFORMATION}

\section{Corresponding Author}

*arlos.marti@uv.es

\section{ACKNOWLEDGMENT}

This work was supported by the EU (ERC Stg Chem-fs-MOF 714122), the Spanish MINECO (MAT-2014-56143-R \& MDM-2015-0538) and the Generalitat Valenciana (GV/2016/137). C.M.-G. and V.R.-G. thank the Spanish MINECO and MECD for a Ramón y Cajal Fellowship and FPU Scholarship, respectively. J.C.W. acknowledges FCT support (project UID/Multi/04349/2013). We also thank J. A. Carrasco, S. Mañas-Valero for help with the experimental work and J. M. MartínezAgudo for the magnetic measurements and S. Tatay for helpful discussions.

\section{REFERENCES}

(1) Coronado, E.; Day, P. Magnetic Molecular Conductors. Chem. Rev. 2004, 104 (11), 5419-5448.

(2) Clemente-León, M.; Coronado, E.; Martí-Gastaldo, C.; Romero, F. M. Multifunctionality in Hybrid Magnetic Materials Based on Bimetallic Oxalate Complexes. Chem. Soc. Rev. 2011, 40 (2), 473-497.

(3) Abellán, G.; Martí-Gastaldo, C.; Ribera, A.; Coronado, E. Hybrid Materials Based on Magnetic Layered Double Hydroxides: a Molecular Perspective. Acc. Chem. Res. 2015, 48 (6), 16011611.

(4) Maspoch, D.; Ruiz-Molina, D.; Veciana, J. Old Materials with New Tricks: Multifunctional Open-Framework Materials. Chem. Soc. Rev. 2007, 36 (5), 770-818.

(5) Kurmoo, M. Magnetic Metal-Organic Frameworks. Chem. Soc. Rev. 2009, 38 (5), 1353-1379.

(6) Dechambenoit, P.; Long, J. R. Microporous Magnets. Chem. Soc. Rev. 2011, 40 (6), 3249.

(7) Coronado, E.; Mínguez Espallargas, G. Dynamic Magnetic MOFs. Chem. Soc. Rev. 2013, 42 (4), 1525-1539.

(8) Dybtsev, D. N.; Chun, H.; Yoon, S. H.; Kim, D.; Kim, K. Microporous Manganese Formate: a Simple Metal-Organic Porous Material with High Framework Stability and Highly Selective Gas Sorption Properties. J. Am. Chem. Soc. 2004, 126 (1), 32-33.

(9) Zheming Wang; Bin Zhang; Mohamedally Kurmoo; Mark A Green; Hideki Fujiwara; Takeo Otsuka, A.; Hayao Kobayashi. Synthesis and Characterization of a Porous Magnetic Diamond Framework, Co3(HCOO)6, and Its N2 Sorption Characteristic. Inorg. Chem. 2005, 44 (5), 1230-1237.

(10) Wang, Z. M.; Zhang, Y. J.; Liu, T.; Kurmoo, M.; Gao, S. [Fe3(HCOO)6]: a Permanent Porous Diamond Framework Displaying H2/N2 Adsorption, Guest Inclusion, and GuestDependent Magnetism. Adv. Func. Mater. 2007, 17 (9), 15231536.

(11) Guillou, N.; Gao, Q.; Noguès, M.; Morris, R. E.; Hervieu, M.; Ferey, G.; Cheetham, A. K. Zeolitic and Magnetic Properties of a 24-Membered Ring Porous Nickel(II) Phosphate, VSB-1. C.
R. Acad. Sci. 1999, 2 (7-8), 387-392.

Brozek, C. K.; Dinča, M. Cation Exchange at the Secondary Building Units of Metal-Organic Frameworks. Chem. Soc. Rev. 2014, 43 (16), 5456-5467.

Rosi, N. L.; Kim, J.; Eddaoudi, M.; Chen, B.; O'Keeffe, M.; Yaghi, O. M. Rod Packings and Metal-Organic Frameworks Constructed From Rod-Shaped Secondary Building Units. J. Am. Chem. Soc. 2005, 127 (5), 1504-1518.

Caskey, S. R.; Wong-Foy, A. G.; Matzger, A. J. Dramatic Tuning of Carbon Dioxide Uptake via Metal Substitution in a Coordination Polymer with Cylindrical Pores. J. Am. Chem. Soc. 2008, 130 (33), 10870-10871.

Bloch, E. D.; Queen, W. L.; Krishna, R.; Zadrozny, J. M.; Brown, C. M.; Long, J. R. Hydrocarbon Separations in a Metal-Organic Framework with Open Iron(II) Coordination Sites. Science 2012, 335 (6076), 1606-1610.

Cadot, S.; Veyre, L.; Luneau, D.; Farrusseng, D.; Alessandra Quadrelli, E. A Water-Based and High Space-Time Yield Synthetic Route to $\mathrm{MOF} \mathrm{Ni}_{2}$ (Dhtp) and Its Linker 2,5-Dihydroxyterephthalic Acid. J. Mater. Chem. A 2014, 2 (42), $17757-$ 17763.

(17) Bloch, E. D.; Murray, L.J.; Queen, W. L.; Chavan, S.; Maximoff, S. N.; Bigi, J. P.; Krishna, R.; Peterson, V. K.; Grandjean, F.; Long, G. J.; Smit, B.; Bordiga, S.; Brown, C. M.; Long, J. R. Selective Binding of O2 Over N2 in a Redox-Active Metal-Organic Framework with Open Iron(II) Coordination Sites. $J$. Am. Chem. Soc. 2011, 133 (37), 14814-14822.

(18) Wang, L. J.; Deng, H.; Furukawa, H.; Gándara, F.; Cordova, K. E.; Peri, D.; Yaghi, O. M. Synthesis and Characterization of Metal-Organic Framework-74 Containing 2, 4, 6, 8, and 10 Different Metals. Inorg. Chem. 2014, 53 (12), 5881-5883.

Dietzel, P. D. C.; Panella, B.; Hirscher, M.; Blom, R.; Fjellvåg, H. Hydrogen Adsorption in a Nickel Based Coordination Polymer with Open Metal Sites in the Cylindrical Cavities of the Desolvated Framework. Chem. Commun. 2006, 959-961.

(20) Shannon, R. D. Revised Effective Ionic Radii and Systematic Studies of Interatomic Distances in Halides and Chalcogenides. Acta Crystallogr. Sect. A 1976, 32 (5), 751-767.

(21) Biesinger, M. C.; Payne, B. P.; Grosvenor, A. P.; Lau, L. W. M.; Gerson, A. R.; Smart, R. S. C. Resolving Surface Chemical States in XPS Analysis of First Row Transition Metals, Oxides and Hydroxides: Cr, Mn, Fe, Co and Ni. App. Surf. Sci. 2011, 257(7), 2717-2730.

(22) Nakamoto, K. Infrared and Raman Spectra of Inorganic and Coordination Compounds: Part B: Applications in Coordination, Organometallic, and Bioinorganic Chemistry; John Wiley \& Sons, Inc.: Hoboken, NJ, USA, 2008

Greenwood, N. N.; Gibb, T. C. Mössbauer Spectroscopy; Chapman and Hall, Ltd. Publishers: London, 1971.

Park, J.; Kim, H.; Jung, Y. Origin of Selective Guest-Induced Magnetism Transition in Fe/MOF-74. J. Phys. Chem. Lett. 2013, 4(15), 2530-2534.

(25) Lian, X.; Feng, D.; Chen, Y.-P.; Liu, T.; Wang, X.; Zhou, H.-C. The Preparation of an Ultrastable Mesoporous Cr(III)-MOF via Reductive Labilization. Chem. Sci. 2015, 6 (12), 70447048.

(26) Bloch, W. M.; Burgun, A.; Coghlan, C. J.; Lee, R.; Coote, M. L.; Doonan, C. J.; Sumby, C. J. Capturing Snapshots of Post-Synthetic Metallation Chemistry in Metal-Organic Frameworks. Nature Chem. 2014, 6(10), 906-912.

Liu, T.-F.; Zou, L.; Feng, D.; Chen, Y.-P.; Fordham, S.; Wang, X.; Liu, Y.; Zhou, H.-C. Stepwise Synthesis of Robust MetalOrganic Frameworks via Postsynthetic Metathesis and Oxidation of Metal Nodes in a Single-Crystal to Single-Crystal Transformation. J. Am. Chem. Soc. 2014, 136 (22), 7813-7816. 